BACTERIOLOGIE

\title{
Communication
}

\section{Note sur un essai d'utilisation d'un substitut de sérum pour la croissance des mycoplasmes}

\author{
A. Bréard ${ }^{1}$
}

BRÉARD (A.). Note sur un essai d'utilisation d'un substitut de sérum pour la croissance des mycoplasmes. Revue Élev. Méd. vét. Pays trop., 1990,43 (4) : 457-458

Un produit de synthèse (Basal Medium Supplementation, BMS) a été utilisé comme substitut de sérum lors de cultures de mycoplasmes. En milieu gélosé, les colonies deviennent atypiques. En milieu liquide, l'examen microscopique ne montre que des éléments coccoïdes et la croissance est moindre, quantitativement, par rapport aux milieux contenant un sérum animal. Après la dixième subculture, lors d'un passage sur milieu traditionnel, les bactéries retrouvent toutes leurs caractéristiques. Grâce à sa composition connue, ce substitut de sérum devrait pouvoir être utilisé pour des études fines des mycoplasmes (en biologie moléculaire par exemple), mais il n'est pas conseillé pour l'isolement des souches ou la production d'antigènes. Mots clés : Mycoplasme - Milieu de culture - Sérum synthétique.

\section{Introduction}

Les mycoplasmes sont des bactéries qui exigent pour leur croissance des milieux de culture complexes, contenant obligatoirement du sérum animal (veau, cheval ou porc par exemple) en proportion variable ( 5 à 50 p. 100).

Récemment, un substitut liquide de sérum*, remplaçant le sérum de veau foetal dans les cultures cellulaires, a été utilisé par le Laboratoire de Bactériologie de l'Institut d'Élevage de Médecine Vétérinaire des Pays Tropicaux (IEMVT) à la place du sérum de cheval dans les milieux de culture pour mycoplasmes. L'objectif était de tester ce produit de synthèse afin de savoir s'il pouvait favoriser, par rapport au sérum de cheval, la croissance des mycoplasmes. Sa formule est donnée en annexe. II est livré stérile et peut, le cas échéant, être filtré à $0,45 \mu$ et $0,2 \mu$.

\section{Matériel et méthode}

Le milieu de base (gélosé ou liquide) utilisé pour le test est le Heart Infusion Broth ou le Heart Infusion Agar ${ }^{*}$, supplémenté en extrait frais de levure (10p. 100) et en ADN $(1 \mathrm{ml}$ d'une solution à $0,2 \mathrm{p} .100$ dans $100 \mathrm{ml}$ de milieu complet). Le substitut entre pour 15 p. 100 dans la composition finale du milieu.

Après ensemencement, les tubes de bouillon sont placés en étuve à $37^{\circ} \mathrm{C}$, et les boîtes en étuve à $37^{\circ} \mathrm{C}$ en atmosphère humide contenant 7,5 p. 100 de COBINB2. Les repiquages en bouillon ont lieu deux fois par semaine jusqu'à la dixième subculture.

\footnotetext{
1. Institut d'Élevage et de Médecine Vétérinaire des Pays Tropicaux, Service de Pathologie Infectieuse, 10 rue Pierre Curie, 94704 Maisons-Alfort Cedex, France.
}

Reçu le 12.6.1989, accepté le 15.3.1990.

* Société InterMed (réf. SS173 Séromed)

** Difco
Les cinq souches utilisées pour cette expérimentation proviennent de la collection de l'IEMVT et représentent cinq espèces dont les difficultés de croissance sont variables :

- M. mycoides subsp. mycoides SC (souche B17),

- M. sp. souche F38,

- M. agalactiae (souche 7780),

- M. capricolum (souche C. kid),

- M. mycoides subsp. capri (souche Pg3).

\section{Résultats}

Dès le début, les cultures apparaissent nettement en bouillon, à l'exception de la souche F38 qui sera repiquée en " aveugle " tout au long de l'expérience. La souche de M. agalactiae ne produit plus de "films and spots".

A la cinquième subculture, toutes les souches sont repiquées parallèlement sur gélose avec substitut : les colonies apparaissent plus lentement que de coutume. Elles sont en général petites, granuleuses, déformées, et leur centre caractéristique a disparu. Les "films and spots" ne sont pas observés pour $M$. agalactiae. En bouillon, les examens microscopiques en contraste de phase ne montrent plus d'amas ou de filaments, mais de très petites formes coccoïdes isolées.

La $11^{\circ}$ subculture a lieu sur milieu classique contenant du sérum de cheval décomplémenté : en milieu liquide, les cultures reprennent leur aspect classique (Mycoplasma sp. F38 retrouve ses comètes). Les colonies sur gélose ont leur structure habituelle et $M$. agalactiae produit à nouveau des "films and spots". Toutes les souches sont inhibées par la digitonine.

Lors d'un autre essai, portant alors sur une seule souche (M. mycoides subsp. mycoides SC), le test à la digitonine a été effectué lors de la $12^{\mathrm{e}}$ subculture, sur milieu gélosé contenant le substitut de sérum : l'inhibition par la digitonine est nette. A l'occasion de ce même essai, le test d'inhibition de croissance a donné des résultats équivalents à ceux obtenus d'habitude sur milieu classique. Par ailleurs, si l'on remplace le substitut de sérum ou le sérum par une quantité équivalente d'eau déminéralisée, aucune culture n'est enregistrée. La base seule ne suffit donc pas à assurer la croissance du mycoplasme.

\section{Conclusion}

L'intérêt de ce substitut (Basal Medium Supplementation) réside dans le fait que, sa composition étant connue, il peut servir dans des cas d'analyses fines, en biologie moléculaire par exemple. La recherche du cholestérol total par deux méthodes (1) (enzymatique et chromatographique), en comparant des échantillons de sérum de cheval et de sérum de veau foetal, a donné les résultats suivants : 


\section{Communication}

- sérum de veau foetal $=0,8 \mathrm{mM}$,

$\mathrm{MgCl}_{2}-6 \mathrm{HB}_{2} \mathrm{O}: 150$

- substitut de sérum $=0,15 \mathrm{mM}$.

$\mathrm{NaHCO}_{3}: 900$

La faible quantité de cholestérol détectée dans le substitut (environ 15 fois moins que dans le sérum de cheval habituellement utilisé) explique l'apparence suboptimale des cultures observées.

En revanche, le BMS n'est conseillé ni pour l'isolement des souches, ni pour la production d'antigènes en grandes quantités compte tenu de l'aspect atypique des colonies et des cultures en bouillon d'une part, d'une densité de culture inférieure, malgré tout, à celle observée lorsque l'on utilise du sérum naturel, d'autre part.

Enfin, le prix de ce substitut de synthèse est légèrement inférieur au sérum de veau foetal.

\section{Remerciements}

Nous remercions Mme LAFONT, directrice de l'Unité INSERM-U130 (Unité de recherches sur le transport des lipides), pour la recherche et le dosage du cholestérol total dans nos échantillons.

BRÉARD (A.). Note on an assay of a synthetic serum substitute for growing mycoplasmas. Revue Élev. Méd. vét. Pays trop., 1990, 43 (4) : 457 458

A synthetic serum substitute (Basal Medium Supplementation, BMS) has been utilized for growing mycoplasmas. Over agar medium, colonies become atypical. In liquid medium, microscope examination only reveals coccoidal elements, and their growth is inferior in amount to that obtained with a traditional animal serum. After the tenth subculture, the bacteria recover their full characteristics when submitted to a passage on a traditional medium. Due to its known composition, this synthetic serum substitute should be usable for advanced studies of mycoplasma organisms, in molecular biology for instance, but its use for isolating strains or antigen production is not recommended. Key words: Mycoplasma - Culture medium Synthetic serum substitute.

\section{Bibliographie}

1. CHEILLAN (F.), LAFONT (H.), TERMINE (E.), HAMANN (Y.), LESGARDS (G.). Comparative study of methods for measuring cholesterol in biological fluids. Lipids, 1989, 24 (3) : 224-228.

Composition du Basal Medium Supplementation (en $\mathrm{mg} / \mathrm{l}$ )

$$
\begin{aligned}
& \mathrm{NaCl}: 6000 \\
& \mathrm{KCl}: 300 \\
& \mathrm{NaH}_{2} \mathrm{PO}_{4}-2 \mathrm{H}_{2} \mathrm{O}: 150 \\
& \mathrm{CaCl}_{2}: 100
\end{aligned}
$$

D-glucose : 3000

Rouge phénol : 10

L-alanine : 60

L-arginine- $\mathrm{HCl}: 55$

L-asparagine- $\mathrm{H}_{2} \mathrm{O}: 10$

Acide L-aspartique : 60

L-cystine : 30

L-glutamine : 420

Acide L-glutamique : 130

L-glycine : 15

L-histidine- $\mathrm{HCl}-\mathrm{H}_{2} \mathrm{O}: 10$

L-isoleucine : 150

L-leucine : 310

L-lysine- $\mathrm{HCl}: 180$

L-méthionine : 70

L-phénylalanine : 90

L-proline : 20

L-sérine : 80

DL-thréonine : 60

L-tryptophane : 20

L-tyrosine : 50

L-valine : 100

Chlorure de choline : 4

Acide folique : 4

i-inositol : 7,2

Nicotinamide : 4

D-Ca-panthothénate : 4

Pyridoxal- $\mathrm{HCl}: 4$

Riboflavine : 0,4

Thiamine- $\mathrm{HCl}: 4$

* Y 8015, unité $1 \mathrm{mg}$, réf. InterMed 92008015 : cys-met-his-ile-gluser-leu-asp-ser-tyr-thr-cys. Epidermal Growth Factor EGF (AA 2031). KOMORIYA et al., Proc. Natn. Acad. Sci. USA, 1984, 81 : 1351-1355.

Peptides : 500

Facteur de croissance ${ }^{*}: 2,5$

Y 8016, unité $50 \mathrm{mg}$, réf. InterMed 92008016 : gly-his-lys. Liver Cell Growth Factor (LWF). PICKART et al., Nature, 1980, 288 : 715-717. 